\title{
Similar Yet Different: the Structure of Social Networks of Characters in Seinfeld, Friends, How I met Your Mother, and The Big Bang Theory
}

\author{
Similares Embora Diferentes: a Estrutura das Redes Sociais dos Personagens em \\ Seinfeld, Friends, How I met Your Mother, e The Big Bang Theory
}

\author{
Ana L. C. Bazzan ${ }^{1 *}$
}

\begin{abstract}
Network theory has been used to analyze structures of narratives in works of fiction. Indeed, previous works have shed light on issues related to role detection, for instance. However, few comparative works exist that deal with TV shows. Since these shows are very popular, there are several Internet forums that suggest how similar some of them are, mostly by comparing roles or importance of core characters. Is this popular intuition backed by an objective, numerical analysis using tools from network theory? The goal of this paper is to compare four situation comedies (Seinfeld, Friends, How I Met Your Mother, and The Big Bang Theory) that share a lot in common since their characters are friends living in similar, urban, environments, struggling with their daily lives, careers, and so on. Using tools for analyzing social networks, these shows were compared, showing that their structures and the roles of the core characters are fundamentally different. The only measure that proved to be similar among the four shows is entropy of their graphs, especially when computed over the degree distribution. Keywords: Fictional social network - Centrality measures - Entropy in Networks - TV series
\end{abstract}

Resumo: A teoria de redes sociais tem sido utilizada para analisar a estrutura de narrativas em peças de ficção. De fato, trabalhos anteriores jogaram luz em questões relacionadas, por exemplo, à detecção de papéis. Entretanto, há poucos trabalhos que comparam séries de TV. Uma vez que estas séries são bastante populares, há muitos foruns na Internet que sugerem certa similaridade entre eles, a maioria baseada em comparações dos papéis dos personagens principais. É está intuição popular corroborada por uma análise quantitativa e objetiva, usando ferramentas da teoria de grafos? O objetivo deste artigo é comparar quatro comédias (Seinfeld, Friends, How I Met Your Mother, e The Big Bang Theory) que têm muito em comum, uma vez que seus personagens são amigos vivendo em ambientes urbanos similares, em constante luta diária por carreira, etc. Estas séries são comparadas usando ferramentas de análise de redes sociais, mostrando que as estruturas de tais redes, bem como os papéis dos personagens principais são fundamentalmente distintos. A única medida que mostra alguma similaridade entre as quatro séries é a entropia, especialmente quando calculada sobre a distribuição de graus.

Palavras-Chave: Rede social em ficção - Centralidade em redes - Entropia em redes - séries de TV

${ }^{1}$ Inst. of Computer Science, Federal Univ. of Rio Grande do Sul, Brazil

*Corresponding author: bazzan@inf.ufrgs.br

DOI: http://dx.doi.org/10.22456/2175-2745.98367 • Received: 21/11/2019 • Accepted: 15/01/2020

CC BY-NC-ND 4.0 - This work is licensed under a Creative Commons Attribution-NonCommercial-NoDerivatives 4.0 International License.

\section{Introduction}

The use of network theory is receiving increasing attention in the quest for shedding light on questions related to the social network underlying fictional narratives in general. According to [1], "a number of narrative-related problems can be addressed automatically through the analysis of character networks". They explicitly mention summarization, classification, and role detection. However, the analysis of the network of characters can also help draw conclusion about similarities between two or more works of fiction by comparing them. It is obvious that plot, cast, and cinematography play a major role, but also the structure of the network of characters underlying the plot (the social network of the show's plot) can add up to the whole effort by offering some hints.

In the present paper, the particular focus is on comparing four TV situation comedies (sitcoms) that-as far as intuition is concerned-share a lot in common: Seinfeld, Friends, How I Met Your Mother, and The Big Bang Theory. In fact, there 
has been a lot of discussion regarding similarities between, e.g., Seinfeld and Friends, or Friends and How I Met Your Mother. Such discussions take place mostly in Internet forums and are mainly driven by passionate views of fans of this or that show, some of them completely rejecting views of others ${ }^{1}$.

One main question here is then, to what extent the structure of the social networks underlying these shows can add to such discussions. How similar are in fact Friends and How I Met Your Mother, or which lessons and structures did Friends take from Seinfeld? Recall that How I Met Your Mother immediately followed the end of Friends (see Figure 1), whose final episode was watched by 52.5 million viewers, and Friends, on its turn, started when Seinfeld was becoming more and more popular with some innovative formulas, breaking with some sitcom structures. As for The Big Bang Theory, despite the fact that it seems less related to the other three shows due to its focus on science and nerds, there are also forums discussing how some of the characters reminisce characters in Friends.

The fact is that the four shows are pretty much centered on the life of twenty-something urbanites (who, with the progression of the shows, turn into thirty-somethings) in their struggles for life, love, jobs. The characters in each show are friends neither because they interact predominantly in family or office circles (as common in previous sitcoms), nor because they meet regularly at some place such as in a bar. Moreover, one finds several such similarities among subsets of characters (one could even speak of archetypes). For instance, in terms of the job niche, we have from bon vivants with no stable job, or any job for that matter (Kramer, Joey), to successful professionals (Jerry, Barney) or researchers (Ross, Sheldon, Leonard, Raj, and Howard), alongside those trying to combine jobs with their passions (Chandler, Marshall), or still struggling (George, Elaine, Monica, Rachel, Phoebe, Robin, Penny). Worth mentioning also is the presence of on-off relationships between a female and a male in each group of characters.

Table 1 lists some well-known features of the shows, showing which of them are shared. Thus, the question is whether or not these and other similarities somehow correlate with their respective social network structures. How similar are Seinfeld and Friends, or Friends and How I Met Your Mother? Is The Big Bang Theory fundamentally different in terms of relationships of characters?

While, obviously, there are many perspectives upon which one could answer such questions, modern approaches include the analysis about how characters interact. For instance, is there one or two core characters who build up the spinal structure, around which most of the other characters interact? Or, rather, are there some more or less independent cores, which are tied up by means of a reduced set of characters, who act as bridges between such cores? Or is there a more compact network of characters, where almost everybody interacts with

\footnotetext{
${ }^{1}$ Specifically, the hits from the following Google query were investigated: "similarities [between] seinfeld friends "how I met your mother" "big bang theory" ".
}

everybody?

Given such analysis on the structure of the interaction of characters underlying the narratives, how can we shed some light in the debates that go on in Internet forums regarding the aforementioned shows? What can we learn by comparing them?

The first contribution of the present paper is to present a detailled account of how to employ tools from network analysis to answer these questions. Following this, a related contribution is the quantitative comparison of the social network of these shows, in which it is shown that, in fact, several similarities that are always brought up, cannot be backed by the actual figures. Finally, apart from some standard quantitative measures, a new one is introduced, based on entropy, both regarding the whole graphs, as well as regarding the core characters. This way, by means of comparing the entropy with and without each core character, it is possible to relate to other centrality metrics.

The remaining of this paper is organized as follows. In the next section, a brief discussion about other efforts in the direction of using network theory for analyzing TV shows is given. For more details, as well as other references about the use of network theory in fiction, please refer to [1]. Following, the four shows are briefly presented (Section 3). Materials and methods are presented in Section 4. Section 5 then characterizes the social networks of the shows, both at network level and at (core) characters level, with the purpose of using such characterization as a comparison basis among shows.

\section{Related Work}

Beveridge and Shan[2] used network theory to investigate who is/are the most central characters in Game of Thrones. This popular show was also the target of: Jasonov[3] (who computed the importance of characters and used them as features or input to a machine learning algorithm in order to predict how likely to die some characters are); Liu e Albergante[4]; and Stavanja e Klemen[5].

Two sitcoms analyzed in the present work have also received attention in past works: Seinfeld [6] and Friends. In fact, the latter has been the focus of several studies, as follows: Nan, Kim e Zhang[7] used a deep learning model for face recognition in Friends's videos in order to distinguish the six main characters and establish the social network between them. Albright[8] calculated the frequency of characters' shared plotlines (groupings), throughout the entire show, drawing conclusions on who are the most independent characters, and relative importance, for instance. Analyzing the importance of the characters is also the goal in [9]. Seth[10] used the transcripts of Friends available in the Internet, aiming at shedding light on the question about who stood out among the character of the show. The following parameters for each character were accounted for: number of lines and words spoken, number of screen appearances, appearances in some locations, and mentions in the episode title. A similar goal underlies the work in $[11,12]$, where the importance of each 


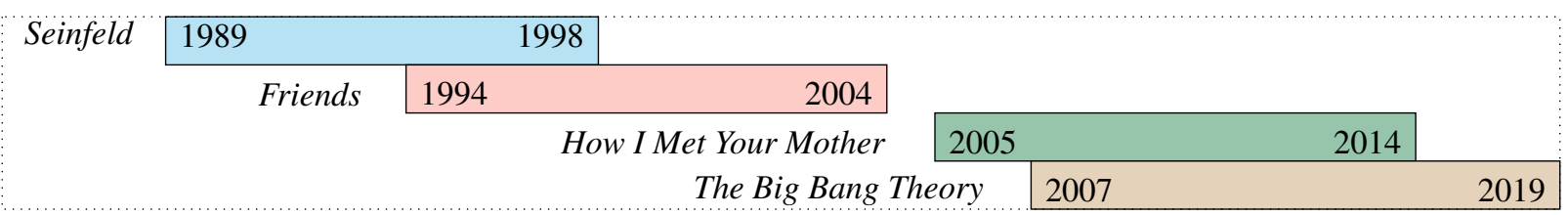

Figure 1. Timeline of the Four Shows.

Table 1. Features Shared by Each Show.

\begin{tabular}{ccccc} 
Shared Feature & Seinfeld & Friends & $\begin{array}{c}\text { Shows } \\
\text { How I Met Your } \\
\text { Mother }\end{array}$ & $\begin{array}{c}\text { The Big Bang } \\
\text { Theory }\end{array}$ \\
\hline $\begin{array}{c}\text { NYC as setting } \\
\text { Apartments across the hall }\end{array}$ & $\sqrt{ }$ & $\sqrt{ }$ & $\sqrt{ }$ & $\sqrt{ }$ \\
$\begin{array}{c}\text { Diner/coffee shop/bar/cafeteria as setting } \\
\text { (high)school friends }\end{array}$ & $\sqrt{ }$ & $\sqrt{ }$ & $\sqrt{ }$ & $\sqrt{ }$ \\
Girl who dates two guys in the group & $\sqrt{ }$ & $\sqrt{ }$ & $\sqrt{ }$ & \\
One character who officiates the marriage of \\
others
\end{tabular}

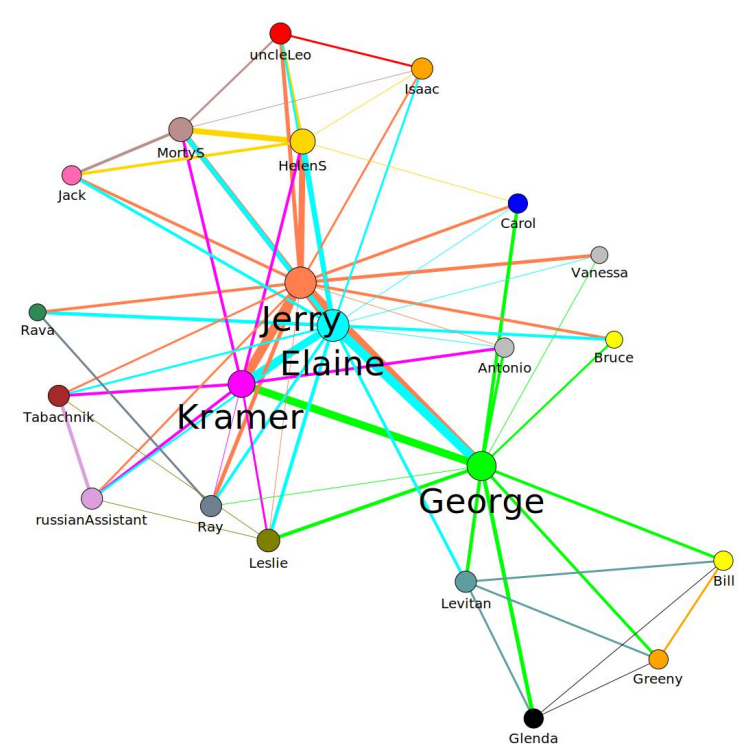

Figure 2. Seinfeld: Network of Characters (22 episodes).

character is investigated using centrality measures. Finally, Edwards et al.[13] compared different extraction methods for Friends using both manually extracted and automated datasets, providing evidence that automated methods of data extraction are reliable for most (though not all) analyses.

Other works, dealing with extraction of interactions (from video, transcripts, etc.) are for instance [14, 15].

These works all investigate specific aspects of individual shows, not aiming at comparisons, with the exception of [16], where the authors have analyzed the character networks of Stargate and Star Trek and found that their structures are similar.

\section{The Four Shows}

\subsection{Seinfeld}

Created by Larry David and Jerry Seinfeld for NBC, Seinfeld is one of the most popular American sitcoms ever. It features Jerry Seinfeld (Jerry Seinfeld), school friend George Costanza (Jason Alexander), friend and former girlfriend Elaine Benes (Julia Louis-Dreyfus), and neighbor across the hall Cosmo Kramer (Michael Richards). Seinfeld is set predominantly in an apartment building in Manhattan's Upper West Side. The "show about nothing" - as often described — began without much fuss in 1989, with a pilot and only 4 other episodes in Season 1 and 12 in Season 2, until reaching the usual number of twenty plus in Season 3, and becoming one of the biggest comedy hits in the U.S. Episodes are about the minutiae of daily life, as experienced by four thirty-something single New Yorkers who had no family responsibilities or other strong responsibilities and hence, allow room for obsessions about small things such as getting a table in a Chinese restaurant, queuing, renting an apartment, finding the car in a parking garage, buying a new suit, getting together with friends, etc.

From beginning, Seinfeld broke several sitcom structures and formulas such a central romantic relationship: Larry David is credited with refusing to focus on a romantic relationship formula between Jerry and Elaine. Rather, episodes would follow a proper structure: the story thread is presented at the beginning, normally involving the characters starting in their own situations; this is then followed by rapid sceneshifts between plot lines bringing the stories together. Thus the characters' stories intertwine in each episode, and, despite the separate plot lines, the narratives maintain the ties among the four characters. This means a change over the usual $\mathrm{A}$ and B plotlines. Besides, the four main characters were not particularly noble human beings. Also, Seinfeld departed from family and group sitcom formulas of its time, in that 


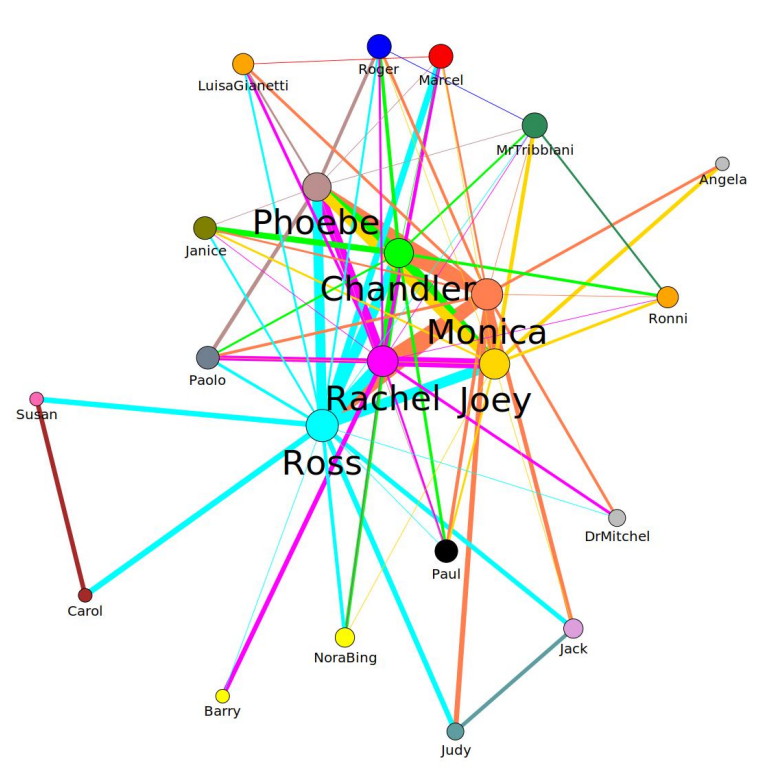

Figure 3. Friends: Network of Characters (22 episodes).

the four main characters were not related by family or work connections, but remain close friends throughout the episodes. Another characteristic of Seinfeld is that a higher than usual number of secondary characters recur and, moreover, play an important role.

All these characteristics have obvious consequences for the social network structure of the show. In fact, as discussed later, characters in Seinfeld have the lowest average degree (number of interactions they are involved in), a clear consequences of the aforementioned separated but tied plotlines. It might be also that the scenes in Seinfeld are longer and thus there are less connections.

\subsection{Friends}

Friends is an American television sitcom created by David Crane and Marta Kauffman, which was aired on NBC from 1994 to 2004. Friends featured six main characters-Rachel Green (Jennifer Aniston), Monica Geller (Courteney Cox), Phoebe Buffay (Lisa Kudrow), Joey Tribbiani (Matt LeBlanc), Chandler Bing (Matthew Perry), and Ross Geller (David Schwimmer), who are friends in their 20s and 30s, living in Manhattan. The story unfolds at three main settings: a Manhattan coffeehouse (Central Perk) and the apartments of Monica and Rachel and Joey and Chandler across the hall. Friends is about young people in a big city coming together to share living expenses, far from parents with new, surrogate family members.

Friends is frequently associated with these facts: (i) all six characters are equally prominent, i.e., they are generally given equal weight across the series; (ii) Friends is a multistory sitcom with no dominant storyline; (iii) Friends is the first true "ensemble" show-a series with no clear star or center, a cast of equals; (iv) the creators of Friends felt that six equal players, rather than emphasizing one or two, would allow for

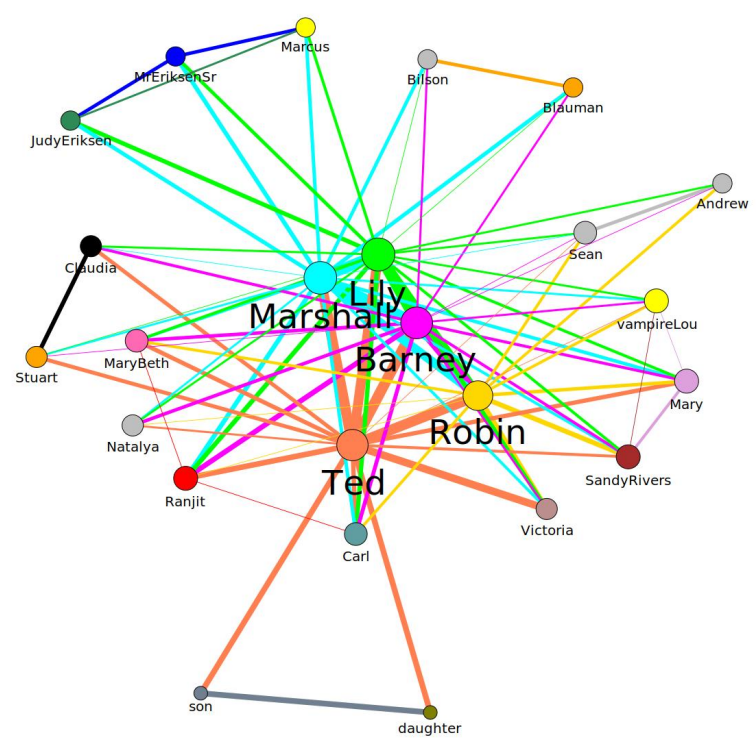

Figure 4. How I Met Your Mother: Network of Characters (22 episodes).

myriad story lines; (v) Monica likes to consider herself as hostess / mother hen; (vi) friendship as surrogate family; (vii) Ross and Rachel have an intermittent relationship.

Most of these facts have a key influence in the structure of the social network. For instance, the facts related to equal weight, multistory, and ensemble character is expected to lead to a less dense social network.

\subsection{How I Met Your Mother}

How I Met Your Mother is an American sitcom created by Craig Thomas and Carter Bays for CBS, featuring as core character Ted Mosby (Josh Radnor) who, in the year 2030, recounts to his children (a daughter and a son) how he met their mother. He goes over nine seasons (208 episodes, from 2005 to 2014) describing the details of the events that led to this encounter, starting in 2005. In this recollection, five other core characters are involved (though the latter appeared only in 28 episodes): Marshall Eriksen (Jason Segel) and Lily Aldrin (Alyson Hannigan) who were friends with Ted since college, the playboy Barney Stinson (Neil Patrick Harris), the TV reporter Robin Scherbatsky (Cobie Smulders), and Tracy McConnell (Cristin Milioti), the mother of the children. Although they all live in Manhattan during the events, they were not all born there. The main settings were apartments in the city, as well as an Irish pub.

The series explores many storylines including the relationship between Marshall and Lily, between Robin and Ted, and Robin and Barney, as well as their career struggles.

How I Met Your Mother has innovated with its non-linear structure as Ted, in the future, recounts the past events from multiple perspectives to the children sitting on a couch, thus exploiting this future-past (in fact present) flashback-flashforward framing in various ways, thus affecting the structure of the 


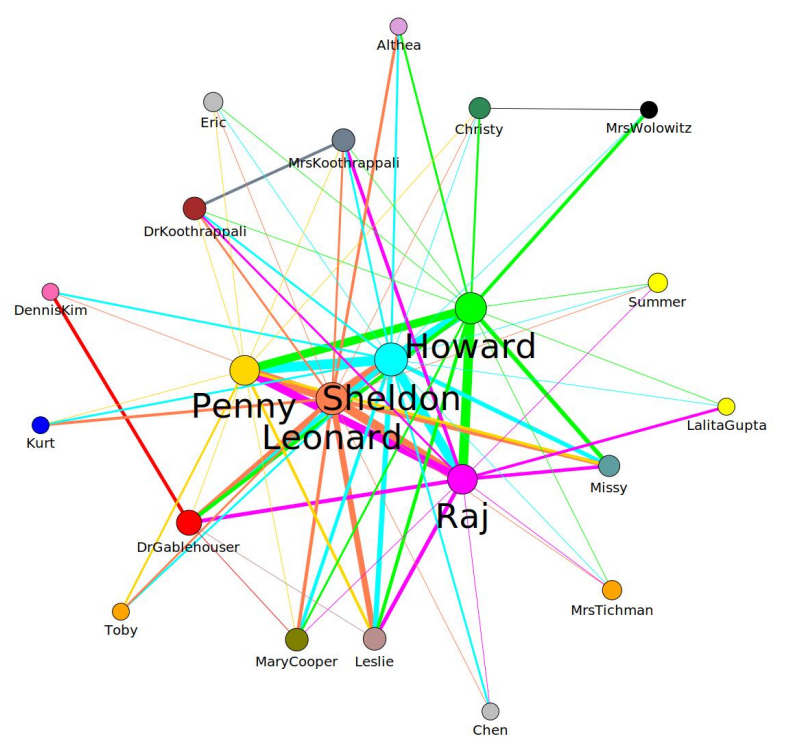

Figure 5. The Big Bang Theory: Network of Characters (22 episodes).

social network.

\subsection{The Big Bang Theory}

The fourth American sitcom featured here is The Big Bang Theory, created by Chuck Lorre and Bill Prady and aired from 2007 to 2019 by CBS. The most prominent characteristic of The Big Bang Theory is that it revolves around science (in particular, physics), being centered on scientists living in Pasadena, where Caltech is located. In its first seasons, it featured five characters: the geeky and socially clueless physicists Sheldon Cooper (Jim Parsons) and Leonard Hofstadter (Johnny Galecki), who share an apartment; the waitress and aspiring actress Penny (Kaley Cuoco), living across the hall; Howard Wolowitz (Simon Helberg), an aerospace engineer; and astrophysicist Raj Koothrappali (Kunal Nayyar). Both Howard and Raj work at Caltech as well. From season four, two other characters get more prominent roles: Bernadette Rostenkowski (Melissa Rauch), a microbiologist who dates and later marries Howard, and the neuroscientist Amy Farrah Fowler (Mayim Bialik), Sheldon's girlfriend.

This show has innovated as well, here not so much by the structure of the narrative, but by featuring characters who depart from the average (wo)men or teenagers in that, as mentioned, the core characters are young scholars with high IQ, having studied in famous universities in the U.S. and with outstanding achievements in science. On the other hand, these characters have social issues like not grasping some social norms (Sheldon), not being able to talk to women (Raj), living with his mother (Howard), and resenting his mother's lack of attention toward him (Leonard). These issues often create innovative situations, in which elaborate forms of communicating scientific facts are perceived as nerdness by other characters such as Penny. Besides, the more intellectual char- acters tend to interact among them.

\section{Materials and Methods}

\subsection{Data Collection and Processing}

Recall that the main objective here is to compare the social network structure of the four shows and draw conclusions about the popular intuition that some of these shows are very similar.

In order to do so, data was manually collected. This was done by the author only, so that the same criteria for extraction of relationships were used. Each episode was watched and notes were taken, regarding how many times who interacts with whom, i.e., based on the actual interactions of characters in each scene of each episode. An interaction happens when two characters talk (even if one talks and the other just listens) or touch or have eye contact. This means that, since not necessarily every character does interact with all others in a scene, each scene is not a complete graph. Thus, there are some differences between the way graphs are constructed using this method and, for instance, methods employed in [2], in [3], and in others, where automated techniques (e.g., language processing) are used to acquire data. The automated way generates complete graphs since all characters in a scene are connected to each other, even if they do not meet (for example, some leave the scene before others enter). As pointed out by Edwards et al.[13], this has an effect in some measures used to perform the analysis. While most of the metrics are not affected by automated data extraction, those related to clustering are not reliable in the automatically extracted networks. These metrics are discussed ahead in this section.

Initially, the idea was to use the first season of each show for such comparison. However, given that there this season varies a lot in number of episodes (see Table 2, where one sees that, in particular, Seinfeld had only five episodes in its first season), the 22 first episodes were considered. This corresponds exactly to Season 1 of How I Met Your Mother, almost the entire Season 1 of Friends, Season 1 plus five episodes of Season 2 for The Big Bang Theory, and, in the case of Seinfeld, the entire seasons 1 and 2 plus five episodes of Season 3. The data is availble at $\langle$ https://github.com/anabazzan/sitcoms $\rangle$.

With such data regarding 22 episodes, interactions among the characters were stored in text files that were then processed using igraph [17] for python. From these files, igraph outputs not only the visualization of the graphs (such as in figures 2 to 5), but also standard metrics that characterize the network, such as number of vertices and edges, average degree, diameter, etc. (see Section 4.1.1). To this, a less usual comparison metric was used, namely entropy (Section 4.1.3).

The goal of such topological characterization of structures within social networks is to try to recognize patterns of interest. Due to the fact that such characterization does not tell much about individual vertices, the present study also considers metrics related to each vertex (i.e., each character) (see Section 4.1.2). Here, not only degree and other centrality 
Table 2. Number of Seasons and Episodes per Show.

\begin{tabular}{ccccc}
\hline Season & Seinfeld & Friends & $\begin{array}{c}\text { Shows } \\
\text { How I Met Your } \\
\text { Mother }\end{array}$ & $\begin{array}{c}\text { The Big Bang } \\
\text { Theory }\end{array}$ \\
\hline 1 & 5 & 24 & 22 & 17 \\
2 & 12 & 24 & 22 & 23 \\
3 & 23 & 25 & 20 & 23 \\
4 & 24 & 24 & 24 & 24 \\
5 & 22 & 24 & 24 & 24 \\
6 & 24 & 25 & 24 & 24 \\
7 & 24 & 24 & 24 & 24 \\
8 & 22 & 24 & 24 & 24 \\
9 & 24 & 24 & 24 & 24 \\
10 & & 18 & & 24 \\
11 & & & & 24 \\
12 & 180 & 236 & 208 & 279 \\
\hline Episodes & & &
\end{tabular}

measures were used, but also one based on what happens with the entropy when key vertices are removed (Section 4.1.4).

In what follows, these metrics are briefly explained. For more details, please refer to [11, 18].

\subsubsection{Standard Metrics Over a Graph}

Metrics that characterize a weighted, undirected graph (network) $G=(V, E)$ as a whole:

- $V$ : set of vertices in the graph $(|V|$ denotes the cardinality of the set or number of vertices)

- $E \subseteq V \times V$ : set of edges (here an edge means a connection between two characters, i.e., an interaction in a scene); edges are weighted by the number of times two characters interact

- Density: ratio of the actual number of edges and the total number of possible edges

- Geodesic shortest path between vertices $i$ and $j$ : path with minimum length that leads from $i$ to $j$

- Diameter: maximum geodesic path in the graph, i.e., maximum distance

- Clique: subset of vertices of a graph, in which any two vertices are directly connected

- Clique number: cardinality of the clique

- Clustering coefficient: measures the ratio of connected triplets (3 vertices fully connected) by the ratio of possible triangles in a graph; it is a measure of how likely two neighbors of a vertex are connected

\subsubsection{Metrics Over a Vertex}

Metrics that characterize a given vertex of the graph in terms of its centrality:

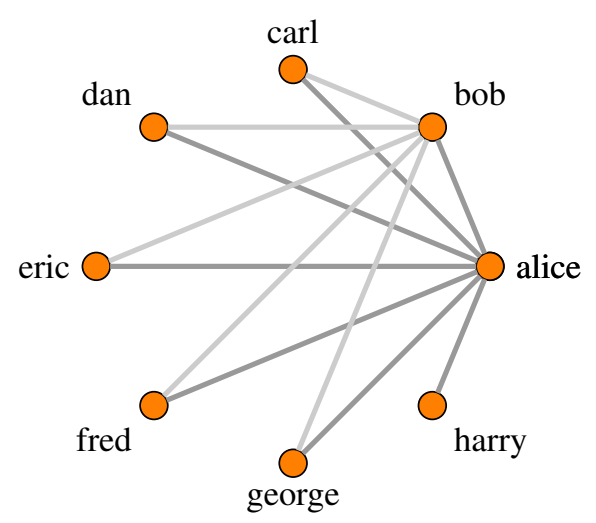

Figure 6. An Example Graph with 8 vertices.

- Degree of vertex $i$ : number of connections a vertex has, including multiple direct connections of $i$ to other vertices

- Betweenness $b_{i}$ of vertex $i$ : number of geodesic paths from vertex $s$ to vertex $t$ that pass through $i$; this quantity can be rescaled by dividing it by the number of pairs of vertices not including $i$ so that $b_{i} \in[0,1]$

- Closeness of vertex $i$ : inverse of the mean shortest distance from $i$ to each of the other vertices

\subsubsection{Entropy to Measure Complexity of a Graph}

The concept of graph entropy was introduced by Rashevsky [19] to assess structural complexity, though entropy also has a root on graph isomorphism and graph invariants. Several graph invariants can be used in the definition of entropy-based measures, and are based on partitions of a graph (also called orbits). One of the most used assessments is called information content regarding a particular aspect of the graph structure, such as the degree distribution. It is based on orbits or partitions formed by the distribution of interest (a graph 
invariant of interest). For instance, if one is interested in the information content $(I)$ regarding the distribution of degrees, the entropy $H$ of a graph $G$ is given by Eq. 1 , where $n$ is the cardinality of $V, n_{l}=\left|V_{l}\right|, V_{l}$ being one of the $k$ subsets of $G$. In each subset, all vertices have the same degree.

$$
I(G)=H=-\sum_{l=1}^{k} \frac{\left|n_{l}\right|}{n} * \log \frac{\left|n_{l}\right|}{n}
$$

In order to compare graphs of different sizes, it is useful to normalize the entropy by dividing Eq 1 by $\log n$. An example graph, partitioned according to degrees distribution, with normalized entropy close to zero would be a star. For instance, take a star with 1000 vertices. One of them has degree 999. All the others have degree 1. Thus, dividing the value from Eq. 1 by $\log (1000)$ would yield a value close to zero. On the other hand, a graph in which all vertices have the same degree has entropy 1. Obviously, several different configurations yield normalized entropy between zero and one. For example, in the graph given in Figure 6, the orbits (partitions) of the graph are: $\{$ carl,dan,eric,fred,george $\}$ (with degree 2 ), $\{$ alice $\}$ (degree 7), \{bob\} (6), \{harry\} (1). Using Eq. 1:

$I=-\frac{5}{8} * \log \frac{5}{8}-3 * \frac{1}{8} * \log \frac{1}{8}$. Thus the normalized entropy would be $\approx 0.67$.

Information content based on degree is not the only possible invariant though. Mowshowitz and Mitsou [20] proposed a method to approximate groups of automorphisms, based on creating a small list of properties of interest, once these can be checked in polinomial time. In short, their procedure is as follows: (1) identify several polynomially checkable properties designed to distinguish between vertices and consider that, in the beginning, all vertices are in the same orbit; (2) for each property and each pair of vertices $u, v$ in an orbit, find whether or not $u$ and $v$ can be distinguished by the property. At the end, if two vertices pass all tests, then they will be considered to be in the same orbit.

By using these procedure, in the present paper, three properties were used to create the partitions of a graph, namely degree, betweenness and closeness. At the end of such procedure, each orbit contains vertices with same degree, same betweenness, and closeness. This way, in Eq. 1, $n_{l}$ is replaced by $X_{l}$, where each $X_{l}$ represents one such partition.

\subsubsection{Assessing the Vitality of a Vertex}

The vitality of a vertex $v$ is defined as the difference of the values yielded by a function $f: G \rightarrow \Re$, when applied on a graph $G$ considering the full set $V$ of vertices, and on a graph without $v$, i.e., on $V \backslash\{v\}$. In particular, in this paper vitality is assessed when $f$ computes the entropy by means of Eq. 1 .

\section{Comparing the Four Shows}

Metrics presented in the last section were computed for the networks of each show. First, those that relate to each graph as a whole (Section 4.1.1) were computed and the results are shown in Table 3. Besides, this table also includes the average degree over all vertices, since this is a further way to characterize the whole graph, even if, as highlighted later, one must be careful when using this for comparisons. Additionally, Table 3 also shows the values regarding the entropies computed over the degree distribution (i.e., each graph's partitions have vertices with the same degree), as well as the distribution in which all vertices of a partition have the same values for the three centralities (degree, betweenness, and closeness).

\subsection{Characterization of the Four Shows Considering Metrics of the Whole Network (Similarities Between Shows)}

In Table 3 one notices a few similarities (in bold): similar number of vertices (characters) in Seinfeld and How I Met Your Mother; and similar number of interactions between Seinfeld and The Big Bang Theory, which give an opportunity for comparing these.

Comparing the first and the last shows in the timelineSeinfeld and The Big Bang Theory, one can see that both have nearly the same number of connections $(|E|)$, in spite of having a very different number of vertices. This way, the densities are very far apart (in fact the former has the lowest value among the four shows, while the latter has the largest value). Note also that the clustering coefficient of The Big Bang Theory is twice that of Seinfeld, and that both have similar values regarding both entropies.

Another partial numerical similarity can be seen between Seinfeld and How I Met Your Mother: both have nearly the same number of vertices (characters) and close clustering coefficient. However, the number of edges (connections) in Seinfeld is much lower.

These two comparisons seem to point out to scenes in Seinfeld lasting longer, what would explain why there are less interactions (recall that interactions are extracted per scene, i.e., if scenes are long then less interactions are recorded).

The two aforementioned comparisons-Seinfeld to The Big Bang Theory and Seinfeld to How I Met Your Motherrefer to (at least partially) numerical similarities that arise when analyzing the whole graphs. However, as mentioned, several internet forums discuss similarities based on the intuition of the fans.

For instance, many people noted various similarities between How I Met Your Mother and Friends (plus, recall that the time interval between both was minimal, as Friends ended May 2004, and How I Met Your Mother started September 2005):

- the setting (NYC)

- an iconic place where they meet (sometimes in the middle of the day!)

- same waiter/bartender throughout the seasons (Gunther and Carl) 
Table 3. Graph's Characteristics of the Four Shows (first 22 episodes); columnwise similarities in bold.

\begin{tabular}{c|ccccccccc}
\hline Graph & $|V|$ & $|E|$ & Density & Diam. & $\begin{array}{c}\text { Clique } \\
\text { Nb. }\end{array}$ & $\begin{array}{c}\text { Clust. } \\
\text { Coef. }\end{array}$ & $\begin{array}{c}\text { Avg. } \\
\text { Degree }\end{array}$ & $\begin{array}{c}\text { Entropy } \\
\text { (degree) }\end{array}$ & (3 centralities) \\
\hline Seinfeld & $\mathbf{1 4 1}$ & $\mathbf{9 1 3}$ & 0.092 & 4 & 8 & $\mathbf{0 . 1 2 4}$ & 12.9 & 0.48 & 0.83 \\
Friends & 114 & 2271 & 0.353 & 5 & 8 & 0.168 & 39.8 & 0.52 & 0.86 \\
How I Met Your Mother & $\mathbf{1 3 7}$ & 1578 & 0.169 & 6 & 8 & $\mathbf{0 . 1 1 9}$ & 23.0 & 0.50 & 0.84 \\
The Big Bang Theory & 60 & $\mathbf{1 0 0 6}$ & 0.568 & 4 & 7 & 0.226 & 33.5 & 0.50 & 0.79 \\
\hline
\end{tabular}

- career women (Rachel and Robin), with poor cooking skills, dating nice nerds (Ross and Ted respectively, who also date their students and love to correct people), and ruin the guys' weddings (with Emily and Stella)

- funny guys (Chandler and Marshall) who met their best friends in college and hate their jobs

- nurturing, controlling "moms" (Monica and Lily), married to the two funny guys, with obsessions (for cleaning and shopping respectively), and fertility woes

- men hooking up with different women (Joey and Barney); both of whom have officiated the weddings of the married couple of the respective show

- the fact that nobody knows or cares about what two characters do for a living (Chandler and Barney)

- some similarities between Phoebe and Barney such as frequently using fake names

- parallels not related to the characters such as the lobster metaphor.

Thus, from the point of view of the social network, how similar are Friends and How I Met Your Mother? Some figures in Table 3 sheds some light. Here too, the values of entropy are very close. On the other hand, it is noticeable that, in the 22 first episodes, there are more characters in How I Met Your Mother than in Friends, which might point out that the former is about a less closed group. Contrarily, there are many more connections in Friends, a much higher density and clustering coefficient. Thus, from the numbers regarding the structure of the networks, these two shows are not very similar. In Section 5.3, it is shown that this changes when it comes to similarities among centrality of characters.

Two other shows for which some similarities seem to exist are Friends and The Big Bang Theory. Table 3 shows that, comparing these two, their social networks have several differences: number of vertices and connections (roughly twice as much for Friends), density and clustering coefficient (much higher for The Big Bang Theory, in spite of this having less vertices and connections). Interestingly, since The Big Bang Theory is very much about the academic world, it is expected that there is a high probability that two characters who are acquainted with the core characters also know each other. This is indeed so, as measured by the clustering coefficient that is higher in The Big Bang Theory as compared to that of Friends

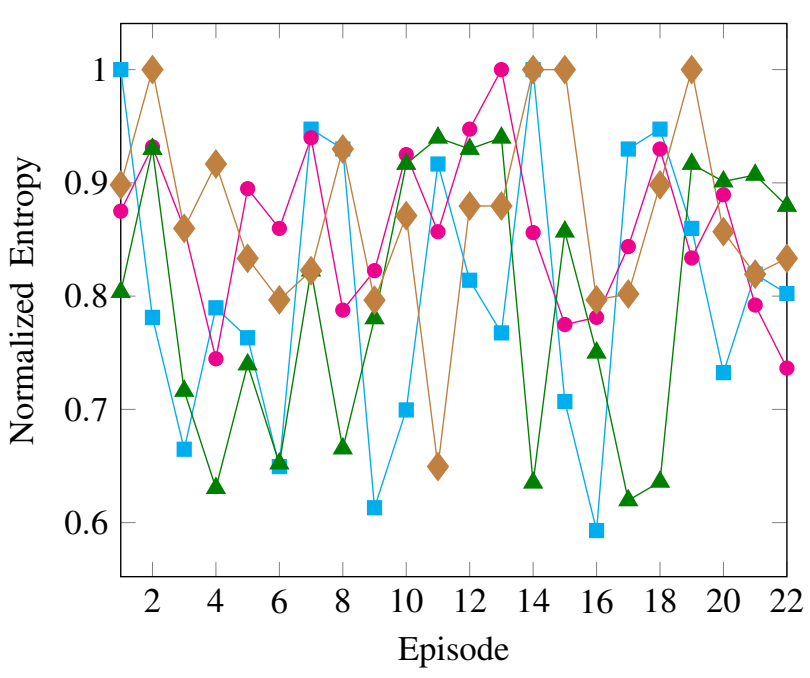

Figure 7. Change in entropy (on degree distribution) for each episode of the four shows (colors follow the previous scheme).

(or to that of any of the other shows as a matter of fact, as seen in Table 3). On the other hand, in Friends, it is well-known that the core characters form a surrogate family, thus other characters almost never meet each other.

Lastly, internet forums also draw lots of comparisons between Seinfeld and Friends, for the reasons discussed before. Taking their social networks into account, what strikes most is that their densities are very different, reflecting the fact that Seinfeld involves many more characters but these build up a number of connections that is less than 50\% that of Friends. This way, the social network of Seinfeld can be said to be very sparse and thus really different from that of Friends.

\subsection{Characterization of the Four Shows Considering their Entropies}

As observed in Table 3, the values of the entropy for each show does not change much - no matter if over degree distribution only, or when the three centralities are considered. A note here is that this remains so if entropy is computed over all episodes (and not only over 22).

However, entropy values do change from episode to episode. Figure 7 depicts such change, for the case of degree distribution. One sees that these values fluctuate between 0.6 and 1 . Recall that entropy 1 means that there is an almost uniform distribution of degree values. Interestingly, such situation happens four times for The Big Bang Theory. 


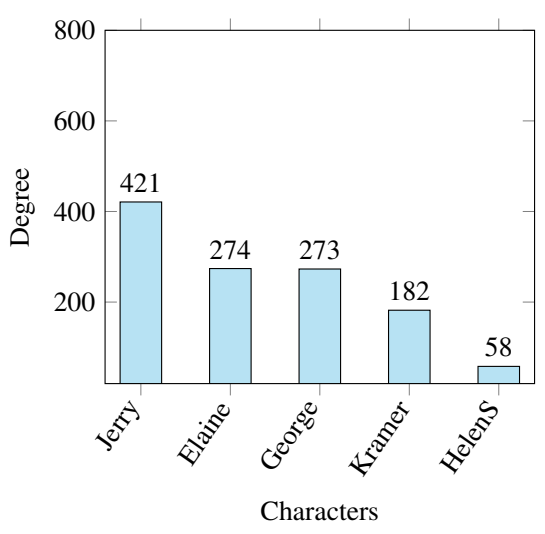

(a) Seinfeld

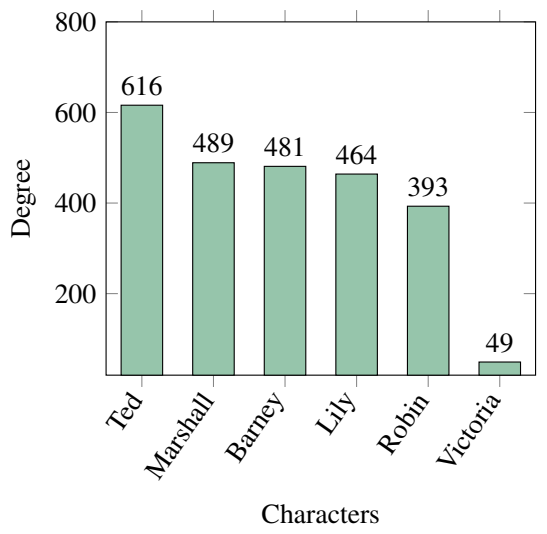

(c) How I Met Your Mother

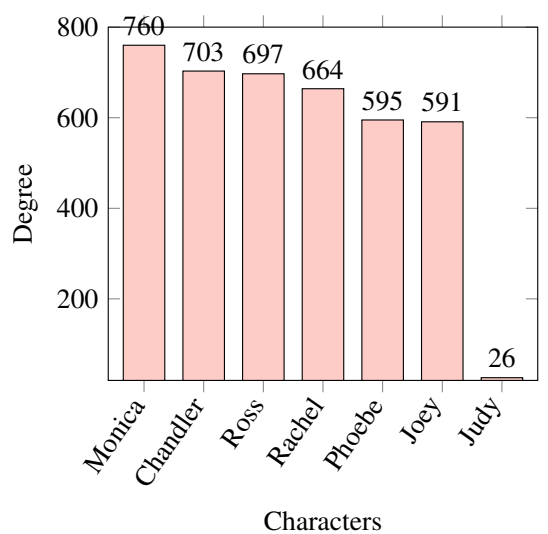

(b) Friends

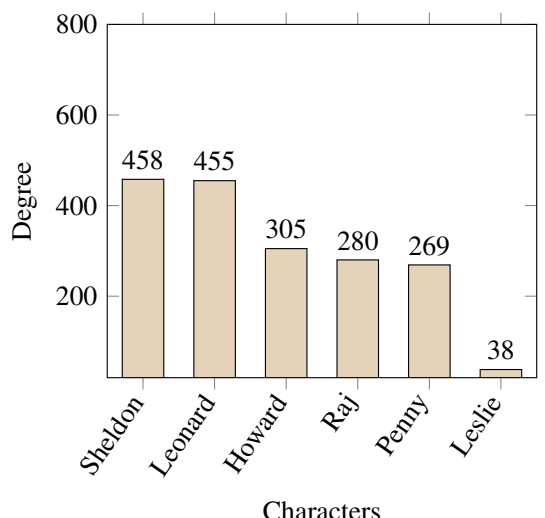

(d) The Big Bang Theory

Figure 8. Degree Centrality of Core Characters (plus one further character)

Going back to the analysis of entropy in the whole graph, the similar values computed for the four shows seem to stem from the fact that the distribution of the centrality measures is very dependent on the core characters, as these represent the bulk of the interactions. Other characters play just a secondary role in the distribution of degrees. Thus the entropy is neither uniform (close to 1), nor has a sharp peak (entropy 0).

To analyse what happens with the core characters only, the entropy of the degree distribution was recomputed when only the core characters' degrees are considered. In this case, the degree distribution of the four core characters of Seinfeld is 0.19 ; it is 0.21 for the six characters of Friends; 0.20 for the five of How I Met Your Mother; and 0.21 for the five in The Big Bang Theory. This then shows that the entropy of the degree distribution is similar for the four sitcoms, no matter if all characters are considered, or only if the core characters are.

\subsection{Characterization of the Four Shows Considering Centrality and Similarity of Individual Charac- ters}

In the previous section, the comparison of the shows was centered on measures that relate to the whole graph (as seen in Table 3). Besides these measures over the whole networks of the four shows, another method to characterize graphs takes the perspective of individual vertices and analyzes how a given measure changes along those elements of the graph. The centrality measures discussed in Section 4.1.2 are good examples. For instance, the degree centrality can point out to how many interactions a given vertex in the network has. A vertex may have a low degree, be connected to other vertices that have likewise low degree, but still be important in the network because it acts as a kind of bridge between groups of vertices. That is the reason why the betweenness centrality is also important. Low values of this quantity mean that a vertex $i$ is separated from other vertices by only a short distance (on average). Thus, in a social network, this vertex would have easier access to information from or influence on other vertices. This is important, e.g., for opinion dissemination, imitation, etc. Likewise, the closeness centrality points out to how close a vertex is from all other vertices. Worth noting also is the fact that other centrality measures are not very discriminating as well $[11,12]$.

As already noted in these two works, closeness varies very little from character to character in Friends. This is also the case for the other three shows. Henceforth, closeness will not be discussed.

In what follows, the comparison is centered in the central- 


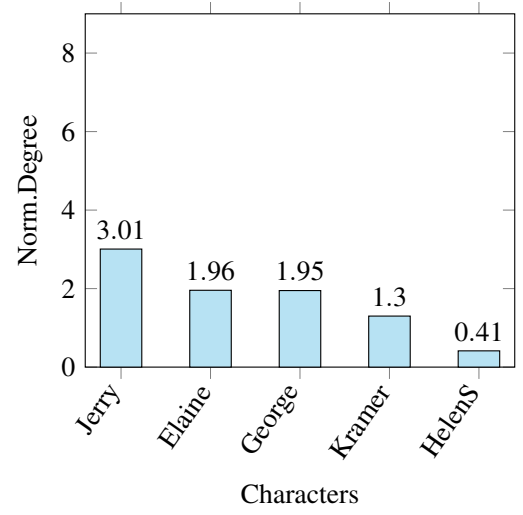

(a) Seinfeld

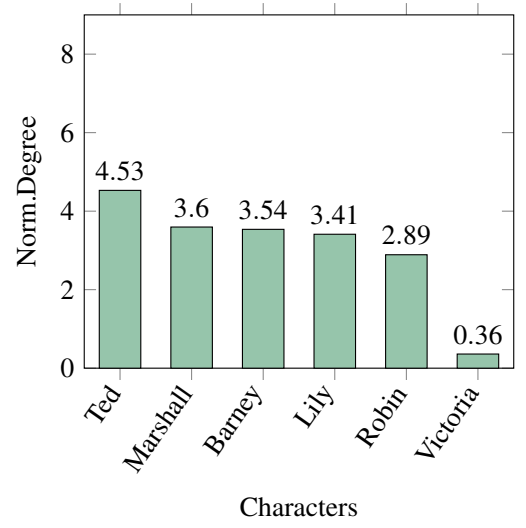

(c) How I Met Your Mother

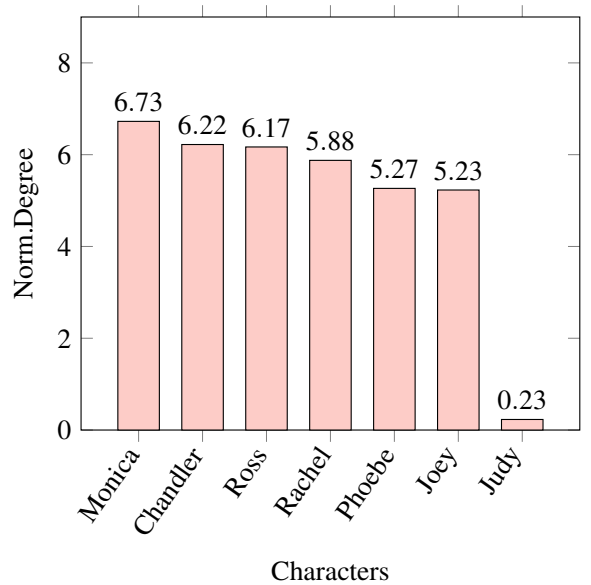

(b) Friends

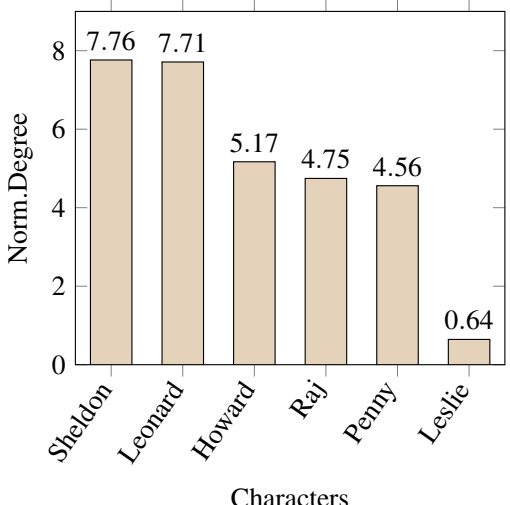

(d) The Big Bang Theory

Figure 9. Degree Centrality (normalized) of Core Characters (plus one further character)

ity measures of individual characters. For this, the core characters of each show are considered. Here, the main centrality measures mentioned before-degree and betweenness-are discussed. Note that, although Table 3 includes the average degree for each sitcom, such values cannot be directly compared, as explained next.

The degree centrality is computed by adding up the number of connections each character has. However, that these values change a lot with the number of vertices $|V|$. For example, a graph $G_{1}$ may have more vertices than a graph $G_{2}$ but, if vertices in $G_{2}$ interact more, then these vertices have higher degree.

Figure 8 depicts the values of the degree centrality for each of the core characters of each of the four shows (plus an additional character who has the next highest degree, for comparison purposes). From there one could conclude that characters in Friends have the highest degrees, followed by those in How I Met Your Mother.

However, from Table 3, the number of vertices in Friends is only the third largest. Thus it is not fair to compare degrees directly as depicted in Figure 8. Rather, it is important to account for the size of the graph. This introduces a further measure, namely, normalized degree, which is obtained by dividing the absolute degree by

$|V|-1$.

Figure 9 depicts normalized degree for each character in each show. It is now possible to draw a fair comparison and conclude that at least some characters in The Big Bang Theory have the highest degrees, as opposed to characters in Friends or How I Met Your Mother. This is remarkable given that The Big Bang Theory has the lowest value of $|V|$, i.e., the least number of characters. It seems then that characters in The Big Bang Theory tend to interact more in most of the scenes, a formula that is difference from the other sitcoms. Also remarkable is the fact that although Seinfeld has the highest value of $|V|$, characters there have the lowest degree (both normalized and absolute). Again, it seems that in Seinfeld there was a trend towards longer scenes, with fewer characters.

Another note regarding degrees: one sees that the additional characters in each plot of Figure 9, i.e., the ones with the next highest degree after the core characters, have degrees that are just a fraction of the others. This is especially the case in Friends, where a well known fact is that the six friends form a closed clique-see [11, 12]. But not only: both Victoria (How I Met Your Mother) and Leslie (The Big Bang Theory) have much lower degrees than the others. In Seinfeld the picture 


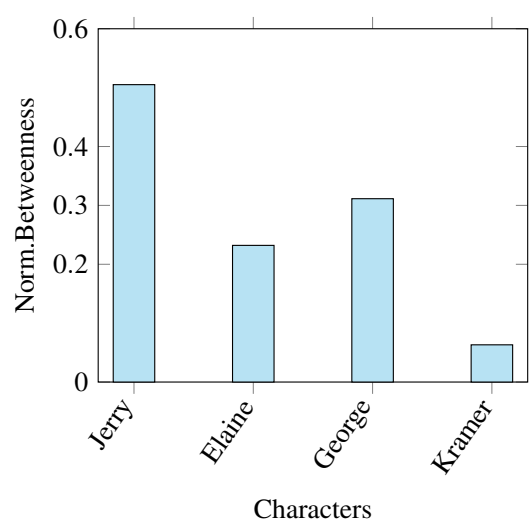

(a) Seinfeld

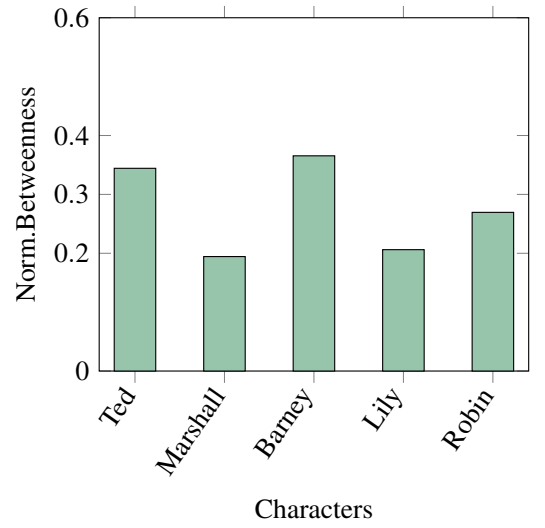

(c) How I Met Your Mother

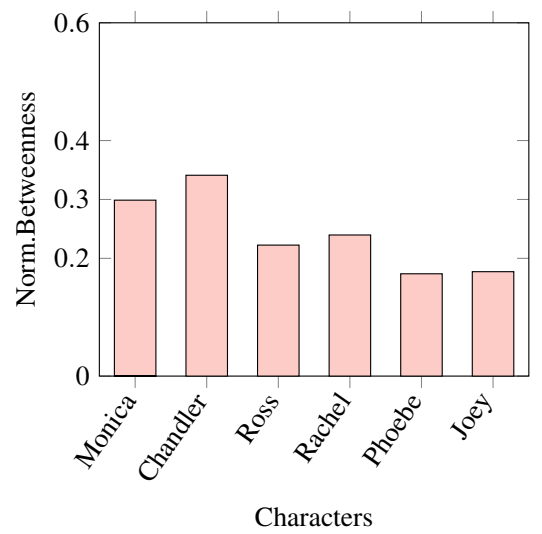

(b) Friends

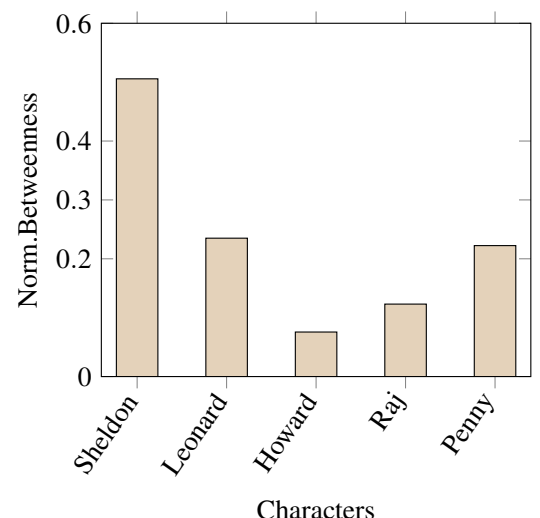

(d) The Big Bang Theory

Figure 10. Betweenness Centrality (normalized) of Core Characters

is a little different since Jerry's mother, Helen Seinfeld, has a degree value that, albeit low, is relatively high compared to the core characters of Seinfeld. By the way, Jerry's father has a degree very similar to Helen.

Finally, the different distributions of degree values seen in Figure 9(a) to (d) point out to important differences in the shows. In Seinfeld it is clear that Jerry is pivotal as his degree is roughly $50 \%$ higher than those of Elaine and George. Also in How I Met Your Mother it is possible to affirm that Ted is more important than the others. In The Big Bang Theory, Sheldon and Leonard are clearly more central. On the other hand, in Friends, it is another well known fact that the creators of the show intended to have six equally prominent characters. Their degree centrality values indeed reflect this.

In relation to this, it also stands out is that core characters in How I Met Your Mother have the second most uniform distribution of degrees, thus making it more similar to Friends, and thus reinforcing the intuition that these shows share key similarities.

So far the importance of the characters was discussed only in terms of the (normalized) degrees. As mentioned, one character may have low degree but be important in other ways since they connect many others; this importance is given by the betweenness centrality.
Figure 10 plots the normalized betweenness values for core characters. Here, none of the non-core characters have significant betweenness so their values are not shown. In fact, betweenness values of Helen Seinfeld, Judy, Victoria, and Leslie are close to zero.

In Figures 10(b) and (c) it is possible to see a more uniform distribution of betweenness values, as compared to Figure 10(a) and (d). The characters of Friends and of How I Met Your Mother have similar betweenness values, ranging from 0.17 (Phoebe) to 0.34 (Chandler) for Friends-Figure 10(b) and from 0.19 (Marshall) to 0.37 (Barney) -Figures 10(c) while Ted's value is 0.34 , thus reinforcing his central role in the show. In Seinfeld, Jerry's betweenness value is the highest (0.51). This is also the value for Sheldon. Differently from the case considering degree centrality, Leonard's value is not as high as Sheldons when betweenness is considered.

Degree and betweenness centralities were discussed separately. Now, putting them together, it is possible to affirm that each show has its particularities, with no two social networks sharing a lot of similarities. In Seinfeld there is a clear central character (Jerry), both in terms of degree and betweenness. Similarly, Ted is central in How I Met Your Mother although Barney is, not surprisingly, a good connector. In The Big Bang Theory, Sheldon is a good connector to other characters, 
although he pairs up with Leonard in terms of degree. Friends shares none of these characteristics, with the six characters having close degree values, and a more uniform distribution of betweenness, though Chandler has the highest values.

Next, both centrality measures are assessed together, now focussing on how to draw comparisons among all the twenty core characters together, irrespective of in which show they appear. How similar are them?

The intuition behind discussions in Internet forums point out to the following characters being somehow similar:

- Monica and Lily

- Joey and Barney

- Chandler and Marshall

- Elaine, Rachel, Robin, and Penny

- Ross and Ted

- George, Leonard, and Howard

Are they indeed similar? Table 4 shows clusters of characters who have similar degree levels and betweenness levels. These levels were computed as follows: for each show, the corresponding interval between the maximum and the minimum values of normalized degree (respectively normalized betweenness) was partitioned into three bins: low, medium, and high. Then, each character was positioned into one of such bins.

These clusters or groups of characters with same level of degree and betweenness are:

- Jerry, Monica, Ted, Sheldon: high degree and high betweenness

- Kramer, Phoebe, Joey, Lily, Howard, Raj: low degree and low betweenness

- Elaine, George, Rachel: medium degree and medium betweenness

- Chandler and Barney: medium degree but high betweenness

- Ross and Marshall: medium degree but low betweenness

- Robin and Penny: low degree but medium betweenness

Leonard is the only character that does not appear together with others in a such group.

Comparing the popular intuition about similar characters, and the groupings in Table 4, the conclusion is that virtually none of the intuitive similarities are backed by the numbers, at least when the first episodes of each show are investigated. The only exception regards Elaine, Rachel, Robin and Penny, but not fully since they appear in two different
Table 4. Subsets of Characters per Level of Degree and Betweenness Centrality.

\begin{tabular}{|c|c|}
\hline $\begin{array}{l}\text { Norm. } \\
\text { Degree }\end{array}$ & Groups (Subset of Characters) \\
\hline \multirow{3}{*}{$\begin{array}{l}\text { high } \\
\text { medium }\end{array}$} & Jerry, Monica, Ted, Sheldon, Leonard \\
\hline & George, Elaine, Rachel , \\
\hline & Ross, Marshall, Chandler, Barney \\
\hline \multirow[t]{2}{*}{ low } & Kramer, Phoebe, Joey, Lily, Howard, Raj \\
\hline & Robin, Penny \\
\hline $\begin{array}{l}\text { Norm. Be- } \\
\text { tweenness }\end{array}$ & Groups (Subset of Characters) \\
\hline \multirow{2}{*}{ high } & Jerry, Monica, Ted, Sheldon \\
\hline & Chandler, Barney \\
\hline \multirow{2}{*}{ medium } & George, Elaine, Rachel, \\
\hline & Robin, Penny, Leonard \\
\hline \multirow{2}{*}{ low } & Ross, Marshall , \\
\hline & Kramer, Phoebe, Joey, Lily, Howard, Raj \\
\hline
\end{tabular}

groups as Elaine and Rachel have higher centrality than Robin and Penny.

This way, for instance, the two more obvious intuitive comparisons-Monica with Lily, Joey with Barney — cannot be proven by the numbers. Joey, despite sharing a lot in common with Barney, is not as good a connector as Barney, when these 22 episodes are considered. In [11, 12] it was possible to see that Joey's betweenness values change a lot from season to season.

It is also worth noting that some characters who, as long as intuition is concerned, should be highly popular (and hence, have high centralities), do not prove as such. Examples are the career women (especially Robin and Rachel) who, are not among those with high degree or betweenness.

\subsection{Entropy Centrality of Vertices}

In the previous section, an analysis was made, which considered the main centrality measures discussed in the literaturedegree and betweenness. The present section extends this standard analysis by showing which characters are important in terms of their vitalities, as defined in Section 4.1.4.

Figure 11 depicts the values of vitality, where the entropy of the degree distribution is computed with and without each character, in order to assess how vital this character is. Intuitively, the bigger this difference, the more vital, i.e., important, a character is. Note that values in Figure 11 are in the order of $10^{-2}$. This means that the absolute values are small, i.e, the entropy does not change a lot with and without specific character. However, the importance of each is given by the relative values, i.e., by the size of the bars relative to each character. Also, such bars can be compared to those in Figure 9 since those depicts the normalized degrees of each character.

In Figure 11(a) one sees that Jerry is the most vital charac- 
ter, thus remaining the most important. Also, comparing bars in Figure 9(a) and Figure 11(a), the importance order does not change.

In the case of Friends (Figure 9(b) versus Figure 11(b)), the changes in vitalities from bar to bar in Figure 11(b) are greater compared to changes the bars in Figure 9(b), thus pointing to vitality of characters not being as uniform as degree. Also, the vitatily of Rachel, compared to that of Ross, is higher, whereas their degrees were very similar.

A change in importance of characters, as measured by vitality is also noticeable in the case of How I Met Your Mother: whereas Ted has just a slightly higher degree centrality, and the others have roughly the same, the vitality of Barney is almost as high as Ted's. The pattern of vitality of characters in How I Met Your Mother is actually closer to that of betweenness (see Figure 10(c)).

In the case of The Big Bang Theory, there is also a noticeable change, namely the vitality of Howard is smaller than that of others.

In conclusion, for those sitcoms in which there is a main character, the vitality of such characters are the highest. Thus, in this sense, vitality confirms the general trends already seen with degree and betweenness centralities.

\subsection{Characterization of the Four Shows Considering Interactions Among Core Characters}

This section focuses on a last type of comparison, namely the number of interactions characters have in each show, as depicted in Table 5. This table shows, for each pair of characters, the percentage of interactions each pair has. Recall that the total number of interactions in each show, is given in Table 3 under column $|E|$. Since this value varies a lot from show to show, the representation as percentage makes more sense.

The highest percentage of interactions refers to Sheldon and Leonard, followed by Jerry and George, both concentrating above $10 \%$ of the interactions in the respective show. The lowest percentage, $3.4 \%$, occurs in three shows: Seinfeld-between George and Kramer; How I Met Your MotherMarshall and Robin; and The Big Bang Theory-Penny and Raj. In Friends, no two characters have an outstanding number of interactions - again showing the point of equally prominent roles - though some pairs interact slightly less.

Regarding the total percentage of interactions that involves the core characters, one sees that in Seinfeld, the four friends only account for around $40 \%$ of the interactions, whereas this value is $77 \%$ in The Big Bang Theory, and $73 \%$ in Friends. Core characters in How I Met Your Mother account for about $60 \%$ of the interactions. From this point of view, Friends and The Big Bang Theory are similar. However, it is clear that the distribution of interactions among pairs of characters is uneven in The Big Bang Theory, while much more uniform in Friends.

Hence, from this point of view, the four shows are indeed different. While this conclusion was based on the first 22 episodes of each show, preliminary investigations conducted
Table 5. Percentage of Pairwise Interactions Among Core Characters.

\begin{tabular}{rrrr}
\hline \multicolumn{4}{c}{ Seinfeld } \\
& George & Elaine & Kramer \\
\hline Jerry & 10.6 & 9.4 & 7.4 \\
& George & 6.0 & 3.4 \\
& & Elaine & 3.7 \\
& & Total & $\approx 40$ \\
\hline
\end{tabular}

\begin{tabular}{|r|rrrrr}
\multicolumn{5}{c}{ Friends } \\
Monica & Rachel & Chandler & Ross & Joey & Phoebe \\
& 6.5 & 5.9 & 5.9 & 5.2 & 5.8 \\
& Rachel & 4.8 & 5.8 & 4.0 & 5.1 \\
& & Chandler & 5.7 & 5.9 & 5.1 \\
& & & Ross & 5.0 & 4.1 \\
& & & & Joey & 4.0 \\
& & & & Total & $\approx 73$ \\
\cline { 3 - 5 } & & & &
\end{tabular}

\begin{tabular}{rrrrr}
\multicolumn{5}{c}{ How I Met Your Mother } \\
Ted & Barney & Lily & Marshall & Robin \\
& 8.4 & 5.5 & 7.4 & 6.8 \\
& Barney & 4.6 & 5.9 & 4.4 \\
& & Lily & 8.7 & 5.0 \\
& & & Marshall & 3.4 \\
& & & Total & $\approx 60$ \\
\cline { 3 - 4 } & & &
\end{tabular}

\begin{tabular}{|c|c|c|c|c|}
\hline \multicolumn{5}{|c|}{ The Big Bang Theory } \\
\hline \multirow{6}{*}{ Sheldon } & Leonard & Penny & Howard & Raj \\
\hline & 15.7 & 8.5 & 7.9 & 6.9 \\
\hline & Leonard & 8.3 & 8.2 & 7.5 \\
\hline & & Penny & 4.0 & 3.4 \\
\hline & & & Howard & $\begin{array}{r}7.1 \\
\sim 77\end{array}$ \\
\hline & & & Total & $\approx 77$ \\
\hline
\end{tabular}

using more episodes of Friends and The Big Bang Theory confirm this trend.

\section{Main Findings and Concluding Re- marks}

The motivation for this research is the fact that several forums suggest that the four sitcoms considered here have many similarities, both in terms of structure and importance and nature of roles regarding some core characters.

In order to check such intuitions, tools from network theory were used. In particular, the metrics considered were: (i) those that consider number of characters, number of interactions, and other characteristics of the whole network; (ii) metrics that regard centrality of individual characters; and (iii) metrics that assess information content and complexity, by considering the entropy over given distributions, such as the distribution of degrees, and how this changes if a given character is removed from the graph. 


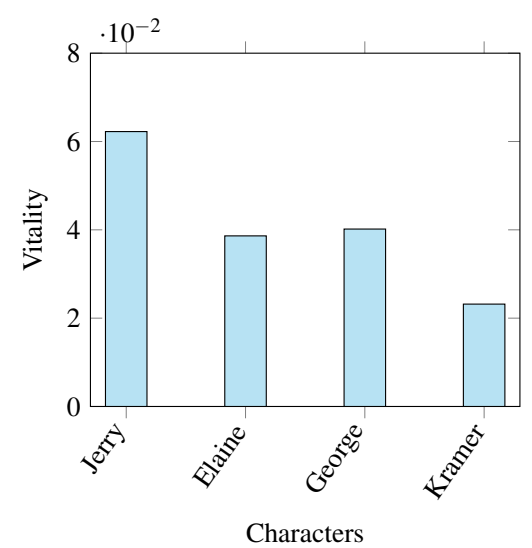

(a) Seinfeld

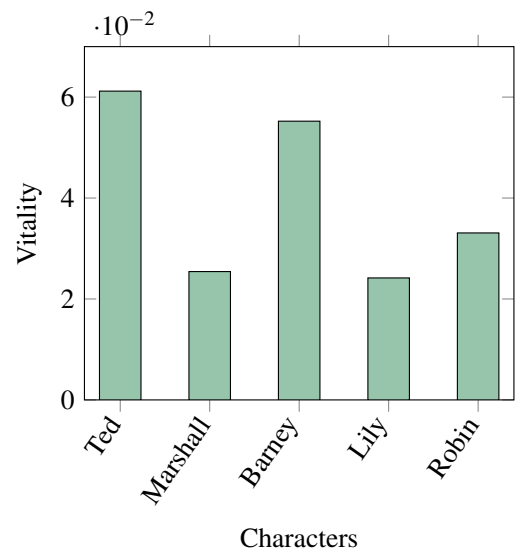

(c) How I Met Your Mother

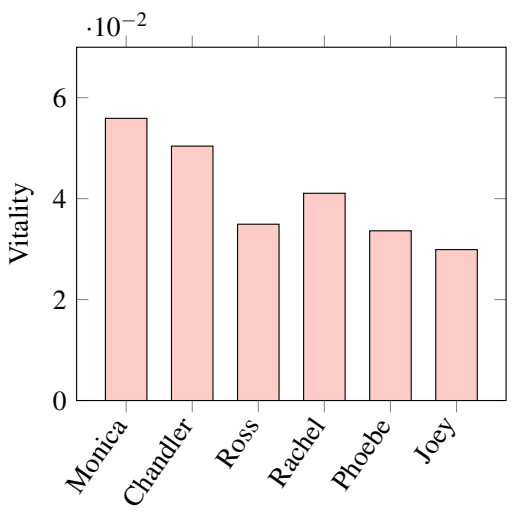

Characters

(b) Friends

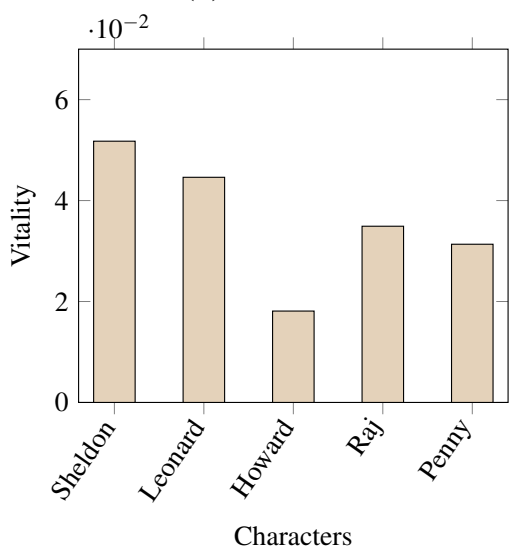

(d) The Big Bang Theory

Figure 11. Vitality Regarding Entropy over Degree Distribution (normalized) of Core Characters

Data of 22 first episodes of the four shows were collected. Pairwise comparisons of two natures were made. First, regarding shows that have either similar number of characters (e.g., Seinfeld and How I Met Your Mother), or similar number of interactions (Seinfeld and The Big Bang Theory). Second, the comparisons included shows that are perceive, in Internet forums, as being similar (e.g., Friends and How I Met Your Mother).

The main conclusions are: (i) despite some shows having similar number of characters, number of connections, or entropies, their densities and/or clustering coefficients are very different; (ii) despite some pairs of shows being perceived as similars by fans in Internet forums, none of them are in fact that similar when those metrics are taken into account.

Following, in sections 5.3 and 5.4, the comparisons were focused on the centrality - degree, betweenness, and vitalityof the core characters. Some similarities were detected here, especially regarding uneven distribution of degrees (in $\mathrm{Se}$ infeld and in The Big Bang Theory) versus a more uniform distribution that is present in Friends and in How I Met Your Mother.

In term of characters, two shows in particular have pivotal characters: Jerry and Ted, though the latter has a degree that is not as high as Jerry's, when the difference to the second highest value is considered. Moreover, Ted's betweenness is not even the highest.

Other facts worth noting regarding betweenness are: (i) the distribution of values, within each show, is uneven, even for Friends's characters; (ii) the range of values is similar for Friends and How I Met Your Mother; (iii) Jerry and Sheldon have the highest values (iv) a few characters who have high degree have low betweenness and vice-versa.

When degree and betweenness centralities are analyzed together, one sees that characters that have similar degree and similar betweenness level belong to the same show. Hence, across different shows, there are no groups of characters that have same centrality pattern. For instance, two always remembered similar characters-Monica and Lily, and Joey and Barney-do not prove that similar as to what regards both degree and betweenness.

Regarding vitality, this metric confirms the others in terms of who are the most prominent characters.

A last comparison was made, by looking at the percentage of pairwise interactions occurring between any two core characters. Two pairwise interactions stand out, namely Jerry and George and Sheldon and Leonard. However, the overall struc- 
ture of interactions and their distributions among the other characters are very different in these two shows. Moreover, while in Seinfeld the core characters are responsible for only $40 \%$ of the total of interactions (thus pointing out to the structure of this show, which involves each character and his/her own network of other acquaintances), this total goes as high as $77 \%$ for The Big Bang Theory, and $73 \%$ in Friends.

In summary, when these four shows are compared using the totality of the metrics, one can conclude that, although there are indeed some similarities, the structure of the shows and the roles of the core characters are fundamentally different when the first episodes of each show are considered. It remains to be investigated which deviations can be noticed when the totality of episodes is considered. Preliminary investigations do point out to the phenomena reported here being a general trend, with few exceptions. A further investigation is planned regarding the temporal aspects, i.e., whether and how the metrics employed here change with the seasons.

\section{Acknowledgments}

Ana Bazzan is partially supported by CNPq (grant 307215/20172 ). She is also grateful for the comments provided by her colleague Prof. Santiago V. Ravelo, which helped improve the process of drawing conclusions regarding the comparison of characters (mainly Table 4).

\section{Author contributions}

This work was developed by the author.

\section{References}

[1] LABATUT, V.; BOST, X. Extraction and analysis of fictional character networks: A survey. ACM Comput. Surv., ACM, New York, NY, USA, v. 52, n. 5, p. 89:1-89:40, September 2019. Disponível em: 〈http://doi.acm.org/10.1145/ $3344548\rangle$.

[2] BEVERIDGE, A.; SHAN, J. Network of thrones. Math Horizons, v. 23, n. 4, p. 18-22, 2016.

[3] JASONOV, M. Network Science Predicts Who Dies Next in Game of Thrones. 2017. Disponível em: $\quad\langle$ https://cns.ceu.edu/article/2017-07-08/ network-science-predicts-who-dies-next-game-thrones $\rangle$. (accessed Jan. 9, 2018).

[4] LIU, D.; ALBERGANTE, L. Balance of thrones: a network study on 'Game of Thrones'. 2017. Disponível em: $\langle$ https://arxiv.org/abs/1707.05213〉. (Accessed August, 2019).

[5] STAVANJA, J.; KLEMEN, M. Predicting kills in Game of Thrones using network properties. 2019. Disponível em: $\langle$ https://arxiv.org/abs/1906.09468〉.

[6] STOLTZMAN, S. Seinfeld Characters - A Post About Nothing. 2016. Disponível em: 〈https://www.stoltzmaniac. com/seinfeld-characters-a-post-about-nothing/ $\rangle$. (Accessed August, 2019).
[7] NAN, C.-J.; KIM, K.-M.; ZHANG, B.-T. Social network analysis of TV drama characters via deep concept hierarchies. In: Proceedings of the 2015 IEEE/ACM International Conference on Advances in Social Networks Analysis and Mining 2015. New York, NY, USA: ACM, 2015. (ASONAM '15), p. 831-836. Disponível em: 〈http://doi.acm.org/10.1145/ 2808797.2809306).

[8] ALBRIGHT, A. The One With All The Quantifiable Friendships. 2015. Disponível em: $\quad\langle$ https://thelittledataset.com/2015/01/20/ the-one-with-all-the-quantifiable-friendships/ $\rangle$. (Accessed August, 2019).

[9] SIMCHONI, G. The one with friends. 2017. Disponível em: 〈http://giorasimchoni.com/2017/06/04/ 2017-06-04-the-one-with-friends/ $\rangle$. (Accessed August, 2019).

[10] SETH, Y. Who was the lead character in Friends? The Data Science Answer. 2017. Disponível em: 〈https://yashuseth.blog/2017/12/29/ data-analysis-lead-character-of-friends-data-science/ $\rangle$.

(Acessed Dec. 30, 2018).

[11] BAZZAN, A. L. C. I will be there for you: six friends in a clique. ArXiv e-prints, April 2018. Disponível em: 〈https: //arxiv.org/abs/1804.04408>.

[12] BAZZAN, A. L. C. I will be there for you: clique, character centrality, and community detection in Friends. Computational and Applied Mathematics. Under review.

[13] EDWARDS, M. et al. The one comparing narrative social network extraction techniques. 2018. Disponível em: 〈http: //arxiv.org/abs/1811.01467〉.

[14] Lv, J. et al. StoryRoleNet: Social network construction of role relationship in video. IEEE Access, v. 6, p. 25958-25969, 2018. ISSN 2169-3536.

[15] BOST, X. et al. Extraction and analysis of dynamic conversational networks from tv series. In: Social Network

Based Big Data Analysis and Applications. [S.1.]: Springer International Publishing, 2018. p. 55-84.

[16] TAN, M. S. A.; UJUM, E. A.; RATNAVELU, K. A character network study of two Sci-Fi TV series. AIP Conference Proceedings, v. 1588, n. 1, p. 246-251, 2014.

[17] CSARDI, G.; NEPUSZ, T. The igraph Software Package for Complex Network Research. InterJournal, Complex Systems, p. 1695, 2006.

[18] COSTA, L. da. F. et al. Characterization of complex networks: A survey of measurements. Advances in Physics, v. 56, n. 1, p. 167-242, 2007.

[19] RASHEVSKY, N. Life, information theory, and topology. The bulletin of mathematical biophysics, p. 229-235, 1955.

[20] MOWSHOWITZ, A.; MITSOU, V. Entropy, orbits, and spectra of graphs. In: ___. Analysis of Complex Networks: From Biology to Linguistics. [S.1.]: Wiley, 2009. cap. 1, p. $1-22$. 